\title{
Bardet Biedl Syndrome: Insights for the Oral Healthcare Provider
}

\author{
Prabhakar Gupta ${ }^{1}$, Tanu Gupta ${ }^{2}$, Pavan Manohar Patil*1 and Seema Pavan Patil ${ }^{3}$ \\ ${ }^{1}$ Department of Oral and Maxillofacial Surgery, School of Dental Sciences, India \\ ${ }^{2}$ Department of Prosthodontics, School of Dental Sciences, India \\ ${ }^{3}$ Consultant Pedodontist and Preventive Dentist, COSMOZONE Dental and Implant Clinic, India
}

*Corresponding author: Pavan Manohar Patil, Professor, Department of Oral and Maxillofacial Surgery, School of Dental Sciences, Greater Noida, Uttar Pradesh-201308, India

\section{ARTICLE INFO}

Received: 幽 December 17, 2020

Published: 慧 December 23, 2020

Citation: Prabhakar Gupta, Tanu Gupta, Pavan Manohar Patil, Seema Pavan Patil. Bardet Biedl Syndrome: Insights for the Oral Healthcare Provider. Biomed J Sci \& Tech Res 32(5)-2020. BJSTR. MS.ID.005304.

\begin{abstract}
Rare diseases pose a particular challenge in provision of oral health care. Most do not have standardized guidelines for oral health care management. This lacuna is magnified in diseases that exhibit dental morphologic anomalies in addition to other systemic findings. Bardet-Biedl syndrome (BBS), a nonmotile ciliopathy of monogenic, autosomal recessive character, is one such disease that has remained an enigma for the oral health care provider. The fact that BBS patients exhibit systemic comorbidities involving the endocrine, renal, respiratory, genitourinary, ophthalmic and cardiovascular systems, as well as cognitive disorders and obesity, a broad spectrum of oral manifestations may be encountered. This review encompasses the insights into the disease, its ethology, pathophysiology, systemic and oral manifestations, diagnosis, medical management and oral health care provision guidelines. The oral health care professional stands a better chance at providing effective and safe oral health care to BBS patients armed with this state-of-the-art knowledge and update.
\end{abstract}

Keywords: Bardet-Biedl syndrome; Etiology; Maxillofacial Manifestations; Dental Management; Ciliopathies; Craniofacial Syndromes

\section{Introduction}

Bardet-Biedl syndrome (BBS) is an autosomal recessive genetic disorder of pleiotropic nature. It exhibits a vast genetic heterogeneity, wherein 21 causal mutations of 21 genes have been identified till date [1]. It is a complex disease involving multiple organ systems such as the renal, endocrine, respiratory, genitourinary, ophthalmic and cardiovascular systems, as well as cognitive disorders and obesity. BBS is classified under ciliopathies, a genetic disorder that affects cellular cilia, their function, or the basal bodies of cells [1]. The cilia are cellular organelles that can be found on the surface of almost all eukaryotic cells and assist in sensory perception and cell motility [2]. Primary cilia have the role of sensing and transducing extracellular signals to control many cellular, developmental and physiological processes in the human body. Therefore, cilia regulate and coordinate several intracellular signalling pathways involved in vertebrate development (stem cells) and tissue homeostasis, while also acting as secretory organelles [2]. Therefore, the fact that cilia are widely expressed in mammalian tissues impresses upon the logical conclusion that ciliary defects would result in several developmental defects and diseases.

BBS has been assigned a code number of \#209900 in the Online Mendelian Inheritance in Man [OMIM] database [3]. It was initially popular as Laurence-Moon-Bardet-Biedl syndrome, owing its name to John Laurence and Robert Moon who first described it in 1866 [3]. Since paraplegia was observed in such patients with absence of obesity or polydactyly (features of BBS), Laurence-Moon syndrome (LMS) and BBS are now considered separate clinical entities [3]. Improved understanding of craniomaxillofacial and dental anomalies associated with BBS is essential to differentiate this entity from other genetic disorders, devising an appropriate oral health care plan and due consultations with the medical care professionals to be able to provide safe and effective oral health 
care to such patients. This review therefore aims to update current knowledge on BBS and provides clinical practice guidelines for oral healthcare management of such patients.

\section{Epidemiology}

BBS is a rare disorder, with a male to female ratio of 1.3:1 [3]. The current prevalence of this syndrome in North America is $1: 100000$, Switzerland is $1: 160,000$ and Spain is $1: 400000$ live births $[3,4]$. However, a higher frequency of BBS is observed in areas with a high rate of consanguinity such as Kuwait $(1: 13,500)$, or from isolated regions such as Newfoundland $(1: 17,500)$ or Faroe Islands $(1: 3,700)[3]$.

\section{Etiopathology}

The primary cilium of human cells performs functions such as modulation of developmental pathways, transduction of sensory signals, alteration of energy homeostasis, and regulation of stem cell proliferation and growth [1]. BBS represents an improper structure or functioning of the primary cilium or nonmotile cilium. The crucial ciliary infraglabellar transport (IFT) proteins are controlled by encoding genes that are affected due to genetic mutations, resulting in manifestation of most craniofacial phenotypes observed in BBS. The IFT protein system controls bidirectional movement along the microtubules of the cilia [1]. The development of various organs in the body, including the craniofacial complex, requires intricate interaction among various tissues, including the neural crest cells, neural ectoderm and surface ectoderm [5]. Murine study results have indicated that loss of primary cilium and ciliary proteins in these tissues during embryonic development gives rise to the development of distinct phenotypes and alterations in cellular differentiation, proliferation, and apoptosis [1].

Structures in the human face largely originate from neural crest cells which are a multipotent cell type derived from the junction of the neural plate and surface ectoderm during early developmental stages. Craniofacial morphogenesis depends upon the controlled migration of these neural crest cells along predefined paths in the neural tube during embryonic development. Therefore, any disturbance in this sequence of events would result in development of a wide spectrum of craniofacial anomalies [6]. A similar pathogenesis could be attributed to dental anomalies in BBS patients. Hisamoto [7] have demonstrated the presence of primary cilia in foetal tooth germs, dental follicle, neonatal tooth germs, adult molar teeth and periodontal ligament. The Shh and Wnt pathways and their interplay is a crucial step in tooth development [1]. Wnt signalling is affected by the loss of IFT ciliary protein kif3a, which results in loss of the Shh pathway, primary controller of amelogenesis and dentinogenetic during tooth formation. This is a direct result of aberrant dental epithelium signals [8]. Hypodontia, observed in a subset of BBS patients, has been suggested to be a result of defective ciliary structure [9].
Celiogenesis in odontoblasts involves the role of BBS4 and BBS6 genes during tooth development, and gene mutations result in tooth abnormalities [10]. Etiology of BBS stems from gene mutations on chromosomes, of which 21 have been identified to date. The chromosomes affected are chromosome 11q13 (BBS 1), 16q13 (BBS 2), 3q11 (BBS 3), 15q22 (BBS 4), 2q31 (BBS 5), 20p12 (BBS 6), 4q27 (BBS 7), 14q32 (BBS 8), 7p14 (BBS 9), 12q (BBS 10), 9q33 (BBS 11), 4q27 (BBS 12), 17q23 (BBS 13), 12q21 (BBS 14), 2p15 (BBS 15), 1q43 (BBS 16), 3p21 (BBS 17), 10q25 (BBS 18), 22q12 (BBS 19), 9p21 (BBS 20) and BBS21 by mutation in the C8ORF37 gene [11]. The BBSome is a protein complex (heteroctameric) known to play a crucial role in primary cilia homeostasis, disturbance of which causes the severe ciliopathies such as BBS [12]. BBS is inherited is an autosomal recessive manner.

\section{Clinical Features}

BBS is a disorder involving multiple organ systems in the body. Most of the symptoms appear progressively during the first decade of life, therefore BBS is predominantly diagnosed in late childhood or early adulthood [13]. High intra and inter familial variability adds to the difficulty in diagnosis of BBS [14]. Primary and secondary clinical features of BBS are enlisted in Table 1 and illustrated in Figure $1[1,3,15]$. Beales et al proposed modified diagnostic criteria in 1999 after reviewing 109 BBS patients [16]. They proposed that a diagnosis of BBS requires the presence of 4 primary features or 3 primaries plus 2 secondary features.

Table 1: Diagnostic clinical features observed in BBS patients.

\begin{tabular}{|c|c|}
\hline Clinical Features & Frequency (\%) \\
\hline \multicolumn{2}{|l|}{ Primary } \\
\hline Retinal Rod-Cone dystrophy & $90-93 \%$ \\
\hline Obesity & $72-90 \%$ \\
\hline Polydactyly & $60-81$ \\
\hline Cognitive impairment/Learning disability & $20-52$ \\
\hline Renal abnormalities & $50-60$ \\
\hline Hypogonadism (in males) & $60-98$ \\
\hline \multicolumn{2}{|l|}{ Secondary } \\
\hline Brachydactyly/syndactyly & $45-100$ \\
\hline Speech delay/disorder & $55-81$ \\
\hline Ataxia & $40-85$ \\
\hline Anosmia/hyposmia & 60 \\
\hline $\begin{array}{l}\text { Dental anomalies (crowding, high arched } \\
\text { palate, hypodontia, small tooth roots) }\end{array}$ & 52 \\
\hline Cardiopathy & 10 \\
\hline Diabetes mellitus & $5-48$ \\
\hline Deafness & $10-12$ \\
\hline
\end{tabular}




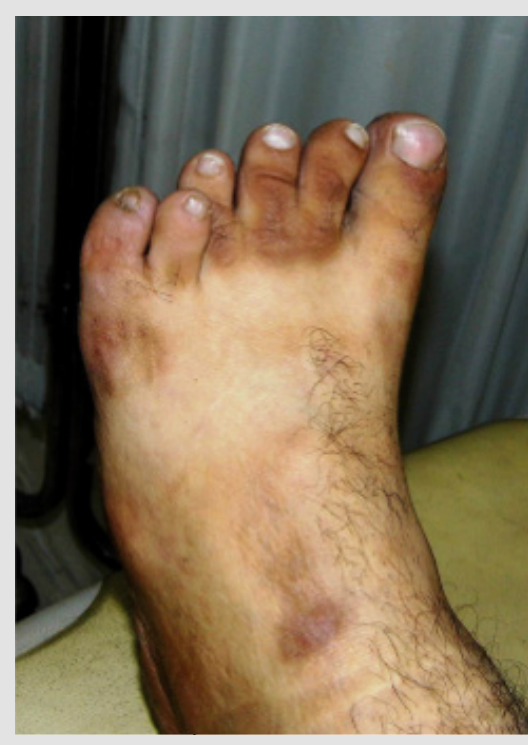

Figure 1: Left foot exhibiting postaxial polydactyly [18].

Destruction of retinal rod cone photoreceptors leads to night blindness (typically by age 5-6 years), constricted visual fields, and eventually complete blindness (median age 15.5 years) [1]. Truncal obesity develops during childhood although birth weight is normal in most BBS patients [3]. Genitourinary abnormalities include horseshoe kidneys, urogenital sinus, vesicoureteral reflux, calyceal, hydrometrical, parenchymal cysts, foetal lobulation, and unilateral renal agenesis [1]. A leading cause of morbidity and mortality in BBS is chronic kidney disease (CKD) [17]. CKD may in the long-term progress to end-stage renal disease that requires dialysis or renal transplantation. Renal function is further affected by the presence of other comorbidities such as diabetes mellitus, hypertension and hyperlipidaemia. Cognitive impairment mainly entails learning difficulties, with a smaller fraction of patients exhibiting IQ impairment, attention deficits, and delayed thought process [3]. The predominant secondary features are enlisted in Table 1. Lesser-known secondary features include developmental delay, behavioural abnormalities, eye abnormalities such as strabismus, cataract or astigmatism, dental anomalies, craniofacial dysmorphism, congenital cardiovascular anomalies and hepatic fibrosis [1].

\section{Orofacial Manifestations}

Orofacial manifestations observed in BBS are listed in Table 2 $[1,3,15,18-20]$. Mouth breathing is observed in about $60 \%$ of patients and could lead to increased anterior facial height, dry mouth, malocclusion, increased dental caries incidence, sleep disturbances and nocturia [1,21]. Incompetent lips may cause difficulty in swallowing due to increased occlusal vertical dimension and malocclusion due to over activity of the perioral musculature [1,22]. Short dental roots or taurodontism may be observed. Figure 2A Gingival hyperplasia is a feature commonly observed in patients who are us- ing medications such as calcium channel blockers, anticonvulsants and immune suppressants, sometimes causing tooth malposition and delayed tooth eruptions $[3,18]$ Figures $2 B$ \& 2 C.

Table 2: Craniofacial manifestations of BBS.

\begin{tabular}{|c|}
\hline Manifestation \\
\hline EXTRA ORAL \\
\hline $\begin{array}{l}\text { Orbital hypertelorism, strabismus, philtrum discrepancies, hypotonic } \\
\text { upper lip, mouth breathing, retrognathia, incompetent lips, bitemporal } \\
\text { narrowing, ptosis, palpebral fissure discrepancies, down-turned mouth, } \\
\text { brachycephaly, macrocephaly, long ears, frontal balding in males, flat } \\
\text { nasal bridge, maxillary atresia, prominent nasolabial folds }\end{array}$ \\
\hline INTRA ORAL \\
\hline $\begin{array}{l}\text { Hypodontia (typically absence of maxillary/mandibular premolars, } \\
\text { lower lateral incisors) }\end{array}$ \\
\hline $\begin{array}{l}\text { High-arched palate, microdontia, crowding and spacing problems, } \\
\text { anterior teeth with short }\end{array}$ \\
\hline $\begin{array}{c}\text { Dental roots and posterior teeth with obliterated pulp chambers, } \\
\text { taurodontism, unilateral or }\end{array}$ \\
\hline Bilateral posterior crossbite, thick mandible and enamel hypoplasia. \\
\hline
\end{tabular}

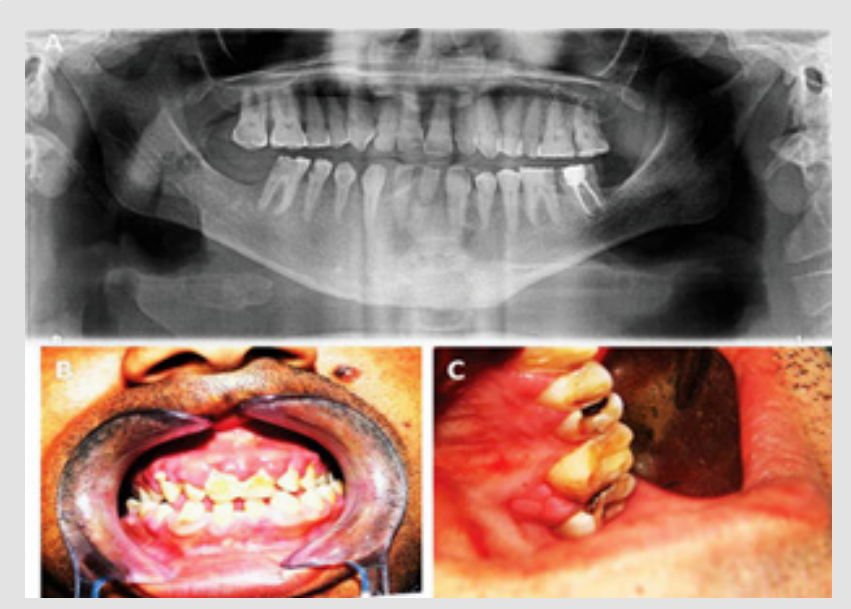

Figure 2A: Orthopantomography showing short dental roots and missing lower lateral incisors.

2B: Gingival enlargement of the upper labial gingivae.

$2 \mathrm{C}$ : Gingival enlargement of the upper palatal gingiva.

Dental crowding is observed in about $46 \%$ of affected individuals, placing them at increased risk of poor oral hygiene, plaque accumulation, periodontitis and halitosis [18,20]. A fraction of patients may exhibit hypodontia, with resultant difficulties in speech, mastication, and phonation. Furthermore, hypodontia related decreased lower facial height and increased overbite may give rise to craniofacial dysmorphism [23]. A high arched palate is observed in about $35 \%$ patients, mainly causing feeding problems. Infants are unable to achieve the required negative pressure to suck breast milk due to poor sucking reflex, thus reflecting in retarded growth and functioning $[3,24]$. Another serious issue in such patients is the compromised airway protection during swallowing which may lead to serious complications due to risk of aspiration of oral contents $[1,24]$. 
Obesity is a predominant feature in patients with BBS, with studies proving that inflammatory processes associated with the condition contribute to development of dental caries, periodontal disease and its progression [25]. Diabetes mellitus (DM) affects about $8 \%$ of BBS individuals, placing them at increased risk of periodontal problems. Periodontal destruction is exacerbated when DM is coexistent with chronic kidney disease (CKD). As a reverse effect, poor glycaemic control may result from dissemination of periodontal pathogens. CKD exerts a negative influence on vitamin D metabolism, consequently affecting activity of bone matrix proteins, leading to periodontal tissue destruction [1,26]. Inflamed periodontal tissue in turn responds with an increased inflammatory response, exacerbating CKD and coronary heart disease.

BBS patients undergoing dialysis therapy or who have undergone renal transplantation commonly manifest hyposalivation or xerostomia, oral candidiasis, uremic fetor and stomatitis, alteration in taste, plaque accumulation, bleeding gums, periodontitis, tooth mobility, petechiae, ecchymosis, enamel hypoplasia, pulp obliterations, demineralization of bone, tooth erosions on lingual surfaces [1,27]. Renal transplant patients commonly present with gingival inflammation because of being treated with immunosuppressive drugs such as cyclosporine $[1,28]$.

\section{Diagnosis}

Apart from the clinical diagnosis based on the Beales et al criteria, currently, diagnostic gene panels are the diagnostic tool of choice [16]. Whole exome sequencing (WES) and whole genome sequencing (WGS) techniques have added a new dimension to genetic testing in BBS due to their ability to discover novel genes [29].

\section{Medical Management}

A multidisciplinary approach is necessary to manage this multifaceted disorder. An annual examination is scheduled to check for eye functions, renal structure and function, blood counts, blood pressure, endocrine functions and psychological wellbeing. There is no specific treatment for BBS, but organ systems are treated based on symptoms. Obese individuals are advised appropriate diet and exercise, visual aids are provided to those visual defects, cognitive disability is addressed through special education, and polydactyly is usually treated with surgery to remove the accessory digit $[3,16]$. The most life-threatening feature of BBS are the renal abnormalities, particularly when renal failure develops, since patients may eventually need dialysis or kidney transplant $[3,30]$.

Currently, gene therapies for BBS, such as gene replacement, readthrough therapy, exon skipping therapy and genome editing are in the developmental stages [29]. Pharmacological interventions such as targeted therapy (melanocortin receptor agonist for obesity control) and drug repurposing (rapamycin for renal cystic phenotype correction) are also undergoing trials [29]. Pharmacogenomic profiling, wherein the effect of a genome on drug response is analysed, may be of significant benefit to BBS patients who may be on multiple medications [29]. Genetic counselling and pre-conception genotyping, as well as prenatal testing for pregnancies at risk and pre-implantation genetic diagnosis seem to be a logical approach to identify the possibility of BBS $[3,16]$. Psychological counselling of the expectant parents could give them a chance to rethink the prospects of a child born with BBS and make an early decision to terminate the pregnancy if they so desire. Those with visual impairment or significant disease status that limits their ability to visit for hospital appointments may benefit from technologies such as telemedicine and online virtual clinics [29].

\section{Dental Management}

Oral healthcare providers must be aware of the perioperative considerations and postoperative complications in obese individuals with BBS, especially regarding the cardiac and respiratory systems [1,31] Armless chairs are suggested in the waiting room as well as the dental operatory. The dental chair and clinic equipment may need recalibration to make them suitable for the obese BBS patient, thereby improving their accessibility to oral health care. Wheelchairs must be available for patients with mobility issues [1,31] Dental caries and periodontal compromise are other findings in obese individuals which need a strict oral hygiene protocol including tooth brushing twice daily, mouthwash and use of dental floss. Regular dental visits for oral prophylaxis and check-up along with a low fat, fibre rich diet should be efficient in oral health maintenance.

Cognitive impairment, learning disabilities or attention deficit disorder may make a BBS child unable to understand verbal cues or exhibit non cooperative or defiant behaviour during dental treatment $[1,18]$. Some children may exhibit autistic-like behaviour and require fixed daily routines to promote function and maintain oral health [18]. Some patients may be afraid and anxiety regarding placement of foreign objects such as toothbrushes or mouthwashes into their mouths, thereby resulting in poor oral health [32]. Patients with cognitive impairment perceive and express dental pain poorly. Presence of dental pain in such patients may be identified only by relative behaviours such as crying, pressing the cheek while eating, difficulty in chewing food or brushing, drooping salivation, putting fingers inside the mouth while eating food and instances of nocturnal earaches $[1,33]$. Parents or guardians must be taught to identify such behaviours and seek immediate treatment. Anxiety in BBS patients may be brought about by a crowded waiting room, dental clinic lights, sounds from air motors or three-way syringes, use of water spray and suction, and the taste of dental materials $[1,34]$. To overcome this situation, a behavioural assessment must be made during a practice visit to a dental clinic so that the patient can familiarize with the dental operatory, its equipment and staff. A routine may be established for such patient which ensures that they take the same route to the dental office, see familiar staff in familiar uniforms, and are treated on the same dental chair every time $[1,34]$. Pediatric dentistry must use a positive reinforcement 
technique wherein children are appreciated for co-operative behaviour $[1,18]$. Tell-show-do technique is of appreciable value in obtaining acceptable behaviour during treatment [35].

Strategies to combat dental anxiety include use of an anxiolytic the night before dental visit, minimizing waiting room time, early morning appointment, soft music, or earplugs to mask the background noise, keeping syringes and other equipment out of patient's vision, and informing the patient before each step of the treatment procedure being performed [1]. 3-D video glasses have been recently suggested to be of great value in audio-visual distraction and calming the patient during dental visits [36]. Dental education of the patient and caregiver could assist in resolving anxiety related apprehensions in the patient and allow the caregiver to better apply home strategies for oral hygiene, while being able to identify symptoms of dental problems [1,34]. Visual problems may seriously compromise oral hygiene practices and access to dental care in BBS patients. Partially visual impaired individuals may benefit from a pre-treatment clinic visit to familiarize themselves [37]. Oral hygiene education and parent counselling may also be undertaken at this visit. Dark eyeglasses must be provided to such patients to avoid acute sensitivity to the dental operating light [37]. When visual impairment is severe, it would be wise to allow parents/caregivers of a visually impaired child/adult to remain in the operatory during the treatment procedure to help relieve dental anxiety and assist in communication [37]. Patients must be clearly instructed about the behaviour expected from them during the procedure and any source of loud noise must be minimized or eliminated as such patients cannot visually define the voices relevant to the source [37].

Verbal description of the sequence of procedural events must be maintained to help visually impaired patients to create a mental picture in their minds. There should not be any sudden jerky movements of the chair, instruments, or the operating dentist [37]. Home oral care includes tooth brushing with soft bristled, multitufted nylon brushes with rounded tips. Electric brushes may prove better than manual counterparts, especially if a caregiver is carrying out the tooth brushing [37]. Dental floss with a holder is recommended for use in plaque control as the tactile perception may assist in placement of the floss in the desired position [37]. The 'Audio tactile performance' (ATP) technique is a recent development, specially designed for visually impaired children. It includes a multisensory health education module creates awareness regarding oral hygiene maintenance [38]. Topical fluoride agents and pit and fissure sealants may be considered to reduce the incidence of dental caries in such patients [37].

Drug induced gingival enlargement in BBS is aggravated by plaque accumulation and mouth breathing [18]. 8-100\% cases exhibit cyclosporine induced gingival enlargement. Factors that determine this condition are the patient's genetic phenotype, age, drug dose and duration, and oral hygiene [18]. Bothersome gingival enlargement requires treatment that may involve gingivectomy, followed by maintenance of oral hygiene and periodontal health. Consultation with the nephrologist is required to plan the gingivectomy and to consider modifying the immunosuppression therapy, particularly when patients experience severe and recurrent drug induced gingival overgrowth [18]. Patients with renal impairment present a special challenge. Uremic stomatitis lesions usually appear on the ventral surface of the tongue and on the anterior mucosal surfaces, typically being very painful in nature [39]. These lesions heal spontaneously in 2-3 weeks following correction of uraemia. In the interim period, topical aesthetic gels containing an antibiotic could be prescribed for symptomatic relief and to prevent infection. Prior to any invasive dental treatment, investigations such as complete blood count and coagulation tests must be obtained, in view of the possible haematological alterations [39]. Any sign of infection in the oral cavity must be eliminated immediately and consideration given to provide antibiotic prophylaxis in procedures that involve bleeding or are at risk of septicaemia [39].

Penicillin's, clindamycin and cephalosporins are the antibiotics of choice and can be administered at their usual doses [39]. However, aminoglycosides and tetracyclines are to be avoided owing to their nephrotoxic potential. Paracetamol is the non-narcotic analgesic of choice for episodic pain, while nonsteroidal anti-inflammatory drugs such as indomethacin, ibuprofen, naproxen and sodium diclofenac are best avoided or prescribed with dose reduction [39]. Narcotic analgesics such as codeine, morphine and fentanyl are metabolized by the liver, and so can be prescribed without dose adjustment [39]. Patients on dialysis need special care. Dental treatment must be scheduled on non-dialysis days, to ensure the absence of circulating heparin, which has a half-life of about four hours [39]. The patient is also in the best physiologic condition with all toxic metabolites cleared from the body. Prior to planned invasive procedures, complete blood count and coagulation tests results must be checked preoperatively. Local haemostatic measures such as mechanical compression, sutures, topical thrombin, microfibrillar collagen and oxidized regenerated cellulose are kept at standby [39]. Tranexamic acid (anti fibrinolytic agent), either as a mouth rinse or systemic administration at a dose of $10-15 \mathrm{mg} / \mathrm{kg}$ body weight a day distributed in 2-3 doses, may also assist in haemostasis [39]. Bacterial endocarditis is a potential complication in dialyzed patients. The current antibiotic prophylaxis regime for infective endocarditis is $2 \mathrm{~g}$ of amoxicillin orally an hour before the procedure. In penicillin allergy patients, cephalexin $2 \mathrm{~g}$, clindamycin $600 \mathrm{mg}$, azithromycin $500 \mathrm{mg}$ or clarithromycin $500 \mathrm{mg}$ are the choices to be orally administered an hour before the procedure [40].

Renal dialysis patients must be analyzed for presence of human immunodeficiency virus [HIV], hepatitis B virus [HBV] and hepatitis $\mathrm{C}$ virus [HCV] since these patients are subjected to numerous blood exchanges and transfusions [39]. In renal transplant patients, all 
dental work must be completed before the transplant. Post renal transplant, oral healthcare providers must avoid dental work on them for 6 months [39]. When invasive procedures are performed, antibiotic cover must be provided. Prolonged corticosteroid therapy predisposes the patient to an adrenal crisis in anxiety provoking procedures such as dentistry. Therefore, doubling the usual dose of steroids on the day of the procedure is considered a safe practice [39]. The most troublesome dental anomalies are short roots and taurodontism. Root canal therapy is often challenging in teeth with short roots. Therefore, it is best to initiate caries preventive measures or treat dental caries at the earliest possible time [41]. Further, such teeth can ill afford loss of supporting structures, making oral hygiene maintenance vital. Taurodontism presents a special challenge about endodontics. Automated root apex locators may be useful to precisely delineate the root apices, while a modified obturation technique combining cold lateral compaction in the apical part with warm vertical compaction in the elongated pulp chamber [42]. It is not recommended to place posts in such teeth to assist in tooth reconstruction [42].

In case of BBS patients requiring operation under a general anaesthetic, such as a facial bone fracture repair or tumour/cyst excision involving the jaws, features such as dental anomalies, high arched palate, facial dysmorphism and morbid obesity may present with significant airway difficulties or impede mask ventilation $[2,43]$. Presence of a bifid epiglottis has been reported to further complicate establishment of a secure airway, wherein use of fibre optic laryngoscopy or video laryngoscopy could prove vital [2]. Gencol [43] emphasized on the fact that ciliary dysfunction leads to thickened airway secretions, thereby making it difficult for the patient to clear the airway after Extubation. They suggested that maintenance of good intra operative hydration would circumvent this problem. Cronje described the first case of BBS with a laryngeal web along with a malacic bifid epiglottis, creating difficulties in intubation and airway management [2]. Fibre optic bronchoscopy is considered the 'gold standard' for an unexpected difficult airway [2].

\section{Conclusion}

Knowledge about rare conditions affecting the orofacial and dental structures is paramount if an oral healthcare provider must provide oral healthcare to affected individuals. Most patients with BBS go unattended because of apathy of the caregiver, lack of knowledge or drive in the oral healthcare provider or the oral healthcare team and severity of comorbidities in such individuals. Collaboration with the medical professionals involved in treating the patient and education of the caregivers are crucial cogs in the wheel of effective and safe oral healthcare provision of patients with BBS. Oral health maintenance is vital to the wellbeing of all patients, especially those with rare diseases, as this goes a long way in improving prognosis and overall life expectancy.

\section{Declarations}

\section{Conflict of Interest: None.}

Funding Source: None.

IRB Clearance: Not applicable.

\section{References}

1. Panny A, Glurich I, Haws RM, Acharya A (2017) Oral and craniofacial anomalies of Bardet-Biedl syndrome: dental management in the context of a rare disease. Journal of Dental Research 96(12): 1361-1369.

2. Cronje L (2017) Bardet-Biedl syndrome: expect the unexpected, suspect the unsuspected. Southern African Journal of Anaesthesia and Analgesia 23(5): 129-138.

3. Álvarez-Satta M, Castro-Sánchez S, Valverde D (2017) BardetBiedl Syndrome as a Chaperonopathy: Dissecting the Major Role of Chaperonin-Like BBS Proteins (BBS6-BBS10-BBS12). Front Mol Biosci 4: 55 .

4. Castro-Sánchez S, Álvarez-Satta M, Pereiro I, Piñeiro-Gallego MT, Valverde D (2014) Algorithm for the molecular analysis of Bardet-Biedl syndrome in Spain. Med Clin (Barc)145: 147-152.

5. Schock EN, Struve JN, Chang CF, Williams TJ, Snedeker J, et al. (2017) A tissue-specific role for intraflagellar transport genes during craniofacial development. PLoS ONE 12(3).

6. Tobin JL, Di Franco M, Eichers E, May-Simera H, Garcia M, et al. (2008) Inhibition of neural crest migration underlies craniofacial dysmorphology and Hirschsprung's disease in Bardet-Biedl syndrome. Proc Natl Acad Sci U S A 105(18): 6714-6719.

7. Hisamoto M, Goto M, Muto M, Nio-kobayashi J, Iwanaga T, et al. (2016) Developmental changes in primary cilia in the mouse tooth germ and oral cavity. Biomed Res 37(3): 207-214.

8. Liu B, Chen S, Cheng D, Jing W, Helms JA (2014) Primary cilia integrate hedgehog and Wnt signaling during tooth development. J Dent Res 93(5): 475-482.

9. Ohazama A, Haycraft CJ, Seppala M, Blackburn J, Ghafoor S, et al. (2009) Primary cilia regulate Shh activity in the control of molar tooth number. Development 136(6): 897-903.

10. Thivichon-Prince B, Couble ML, Giamarchi A, Delmas P, Franco B, et al. (2009) Primary cilia of odontoblasts: possible role in molar morphogenesis. J Dent Res 88(10): 910-915.

11. Manara E, Paolacci S, D Esposito F, Abeshi A, Ziccardi L, et al. (2019) Mutation profile of BBS genes in patients with Bardet-Biedl syndrome: an Italian study. Ital J Pediatr 45: 72.

12. Klink BU, Gatsogiannis C, Hofnagel O, Wittinghofer A, Raunser S (2020) Structure of the human BBSome core complex. Elife 9: e53910.

13. Wormser O, Gradstein L, Yogev Y, Perez Y, Kadir R, Goliand I, et al. (2019) SCAPER localizes to primary cilia and its mutation affects cilia length, causing Bardet-Biedl syndrome. Eur J Hum Genet 27: 928-940.

14. Deveault C, Billingsley G, Duncan JL, Bin J, Theal R, Vincent A, et al. (2011) BBS genotype-phenotype assessment of a multiethnic patient cohort calls for a revision of the disease definition. Human Mutation 32(6): 610-619.

15. Hamosh A (2020) Mc Kusick-Nathans Institute of Genetic Medicine, John Hopkins University School of Medicine.

16. Forsythe E, Beales PL (2013) Bardet-Biedl syndrome. Eur J Hum Genet 21(1): 8-13.

17. Forsythe E, Sparks K, Best S, Borrows S, Hoskins B, et al. (2017) Risk Factors for Severe Renal Disease in Bardet-Biedl Syndrome. J Am Soc Nephrol 28(3): 963-970. 
18. Hassona Y, Kasabreh N, Hammoudeh H, Scully C (2017) Oral healthcare management in Bardet Biedl syndrome. Spec Care Dentist 37(1): 47-50.

19. Andersson EM, Axelsson S, Gjolstad LF, Storhaug K (2013) Taurodontism: a minor diagnostic criterion in Laurence-Moon/Bardet-Biedl syndromes. Acta Odontol Scand 71(6): 1671-1674.

20. Ferreira do Amaral CO, Logar Gde A, Parisi AG, Takahashi K, Straioto FG (2014) General and stomatologic aspects of Bardet-Biedl syndrome. J Craniofac Surg 25(6): e575-e578.

21. El Aouame A, Daoui A, El Quars F (2016) Nasal breathing and the vertical dimension: a cephalometric study. Int Orthod 14(4): 491-502.

22. MacAvoy SK, Jack HC, Kieser J, Farella M (2016) Effect of occlusal vertical dimension on swallowing patterns and perioral electromyographic activity. J Oral Rehabil 43(7): 481-487.

23. Kreczi A, Proff P, Reicheneder C, Faltermeier A (2011) Effects of hypodontia on craniofacial structures and mandibular growth pattern. Head Face Med 7(1): 23.

24. Kudiyirickal MG, Pappachan JM (2015) Diabetes mellitus and oral health Endocrine 49(1): 27-34.

25. Costacurta M, Di Renzo L, Bianchi A, Fabiocchi F, De Lorenzo A, et al. (2011) Obesity and dental caries in paediatric patients: a cross-sectional study. Eur J Paediatr Dent 12(2): 112-116.

26. Fisher MA, Taylor GW, West BT, McCarthy ET (2011) Bidirectiona relationship between chronic kidney and periodontal disease: a study using structural equation modelling. Kidney Int 79(3): 347-355.

27. Bayraktar G, Kurtulus I, Kazancioglu R, Bayramgurler I, Cintan S, et al. (2008) Evaluation of periodontal parameters in patients undergoing peritoneal dialysis or hemodialysis. Oral Dis 14(2): 185-189.

28. Majumdar U, Arya G, Singh S, Pillai A, Nair PP (2012) Oro-dental findings in Bardet-Biedl syndrome. BMJ Case Rep 2012

29. Forsythe E, Kenny J, Bacchelli C, Beales PL (2018) Managing BardetBiedl Syndrome-now and in the Future. Frontiers in Pediatrics 6: 23.

30. Putoux A, Attie-Bitach T, Martinovic J, Gubler MC (2012) Phenotypic variability of Bardet-Biedl syndrome: focusing on the kidney. Pediatr Nephrol 27: 7-15.

31. Krishnan B (2009) Obese oral and maxillofacial surgical patient. J Craniofac Surg 20(1): 53-57.

\section{ISSN: 2574-1241}

\section{DOI: $10.26717 /$ BJSTR.2020.32.005304}

Pavan Manohar Patil. Biomed J Sci \& Tech Res

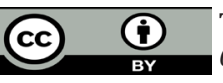

This work is licensed under Creative

Commons Attribution 4.0 License

Submission Link: https://biomedres.us/submit-manuscript.php
32. Shanmugam M, Shivakumar V, Anitha V, Meenapriya BP, Aishwarya S, et al. (2014) Behavioral pattern during dental pain in intellectually disabled children: a comparative study. Int Sch Res Notices 2014: 1-5.

33. Alaki SM, Bakry NS (2012) Dental pain in children with intellectual disabilities: caregivers' perspective. Int J Dent 2012(3): 701608.

34. Dougall A, Fiske J (2008) Access to special care dentistry, part 2. Communication. Br Dent J 205(1): 11-21.

35. Delli K, Reichart PA, Bornstein MM, Livas C (2013) Management of children with autism spectrum disorder in the dental setting: concerns, behavioural approaches and recommendations. Med Oral Patol Oral Cir Bucal 18(6): e862-e868.

36. Nuvvula, S, Alahari, S, Kamatham R (2015) Effect of audiovisual distraction with 3D video glasses on dental anxiety of children experiencing administration of local analgesia: a randomised clinical trial. Eur Arch Paediatr Dent 16: 43-50.

37. Mohan R, Raju R, Gubbihal R, Kousalya PS (2016) Comprehensive dental care for the visually impaired: a review. Int J Oral Health Med Res 3(4): 97-101.

38. Joybell C, Krshnan R, Kumar VS (2015) Comparison of Two Brushing Methods- Fone's vs Modified Bass Method in Visually Impaired Children Using the Audio Tactile Performance (ATP) Technique. Journal of Clinical and Diagnostic Research 9(3):19-22.

39. Jover-Cervero A, Bagán JV, Jiménez-Soriano Y, Poveda-Roda R (2008) Dental management in renal failure: Patients on dialysis. Med Oral Patol Oral Cir Bucal 13(7): E419-426.

40. Thornhill MH, Dayer M, Lockhart PB, Prendergast B (2017) Antibiotic prophylaxis of Infective endocarditis. Curr Infect Dis Rep 19(2): 9.

41. Michelogiannakis D, Vastardis H, Melakopoulos L, Papathanasopoulou C, Tosios KI (2018) The challenge of managing patients with generalized short root anomaly: A case report. Qiuntessense Int 49: 673-679.

42. Nazari S, MirMotalebi F (2006) Endodontic treatment of a Taurodontism Tooth: Report of a case. Iran Endod J 1(3): 114-116.

43. Gencol T, Sergin D, Balcioglu T (2019) Anesthetic Management of a Pediatric Patient With Bardet-Biedl Syndrome: A Case Report. A \& A Practice 12(5): 165-167.

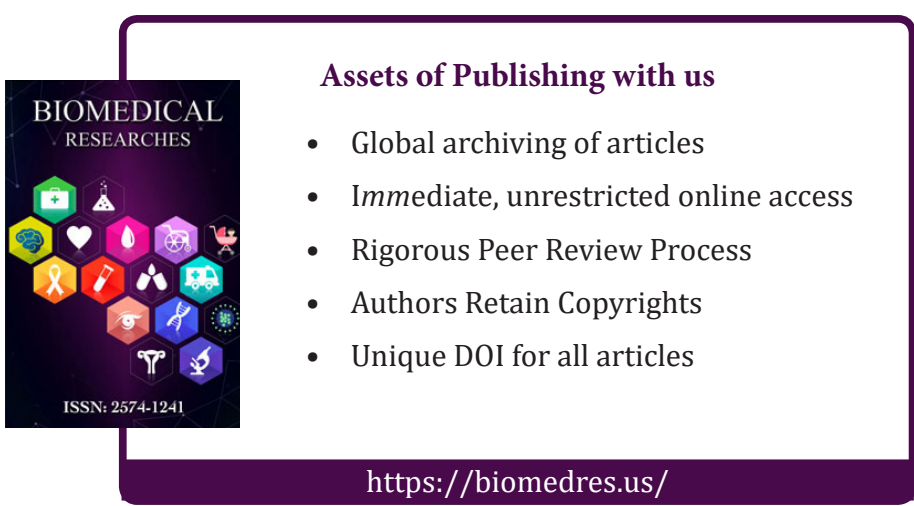

